\title{
Tables of vacuum ultraviolet emission band systems of molecular nitrogen from 82.6 to $124.2 \mathrm{~nm}$
}

J.-Y. Roncin ${ }^{1}$ and F. Launay ${ }^{2}$

${ }^{1}$ Laboratoire Traitement du Signal et Instrumentation (TSI), CNRS UMR 5516, 23 rue du Dr. Paul Michelon, 42023 Saint-Étienne Cedex 2, France

${ }^{2}$ Observatoire de Paris, Section de Meudon, DAMAp and CNRS URA 812, 92195 Meudon Cedex, France

\begin{abstract}
Observed wavenumbers of the emission lines belonging to 10 vacuum ultraviolet band systems of molecular nitrogen are classified into 283 bands, 220 of them reported for the first time ${ }^{1}$.
\end{abstract}

Key words: molecular data - planets and satellites: general

The vacuum ultraviolet (VUV) emission spectrum of molecular nitrogen $\left(\mathrm{N}_{2}\right)$ is of great astrophysical interest. The spectra transmitted from the Voyager 1 and 2 spacecrafts (Broadfoot et al. 1981, 1989; Strobel \& Shemansky 1982) have shown that $\mathrm{N}_{2}$ is the major constituent not only of the Earth's atmosphere, but also of those of Titan and Triton, satellites of Saturn and Neptun respectively. The present paper aims at presenting an extensive set of line wavenumbers for the bands involving transitions toward the electronic ground state $X^{1} \Sigma_{g}^{+}$from 10 singlet electronic excited states of $\mathrm{N}_{2}$, i.e. from the valence states ${ }^{1} \Pi_{u}(b),{ }^{1} \Sigma_{u}^{+}\left(b^{\prime}\right)$, and ${ }^{1} \Pi_{g}(a)$, and the Rydberg states $3 p \pi^{1} \Pi_{u}\left(c_{3}\right), 4 p \pi{ }^{1} \Pi_{u}\left(c_{4}\right), 3 p \sigma{ }^{1} \Sigma_{u}^{+}\left(c_{4}^{\prime}\right), 4 p \sigma^{1} \Sigma_{u}^{+}\left(c_{5}^{\prime}\right)$, $5 p \sigma^{1} \Sigma_{u}^{+}\left(c_{6}^{\prime}\right), 3 s \sigma^{1} \Pi_{u}\left(o_{3}\right)$, and $3 d \pi^{1} \Sigma_{u}^{+}$. In parentheses we have recalled the traditional names of the states. This paper complements another publication by Roncin et al. (1998) where they report the energies for the rovibronic levels of the ten excited states.

The use of a low-pressure Penning-type electric discharge has led to considerably reduced self-absorption at short wavelengths, extending and greatly improving previously published data of Tilford \& Wilkinson (1964). Tables 1 to 10 list all the observed rotational branches of the 283 bands belonging to the 10 band systems $b^{1} \Pi_{u} \longrightarrow X^{1} \Sigma_{g}^{+}$, $b^{\prime}{ }^{1} \Sigma_{u}^{+} \longrightarrow X^{1} \Sigma_{g}^{+}, c_{4}^{\prime}{ }^{1} \Sigma_{u}^{+} \longrightarrow X^{1} \Sigma_{g}^{+}, c_{5}^{\prime}{ }^{1} \Sigma_{u}^{+} \longrightarrow$ $X^{1} \Sigma_{g}^{+}, c_{6}^{\prime}{ }^{1} \Sigma_{u}^{+} \longrightarrow X^{1} \Sigma_{g}^{+}, 3 d \pi^{1} \Sigma_{u}^{+} \longrightarrow X^{1} \Sigma_{g}^{+}$

\footnotetext{
1 Tables 1 to 10 are only available in electronic form at the CDS via anonymous ftp to cdsarc.u-strasbg.fr (130.79.128.5) or via http://cdsweb.u-strasbg.fr/Abstract.html
}

$c_{3}{ }^{1} \Pi_{u} \longrightarrow X^{1} \Sigma_{g}^{+}, c_{4}{ }^{1} \Pi_{u} \longrightarrow X^{1} \Sigma_{g}^{+}, o_{3}{ }^{1} \Pi_{u} \longrightarrow$ $X^{1} \Sigma_{g}^{+}$, and $a^{1} \Pi_{g} \longrightarrow X^{1} \Sigma_{g}^{+}$(Lyman-Birge-Hopfield), respectively. All VUV transitions terminate on the electronic ground state $X^{1} \Sigma_{g}^{+}$and an abbreviated notation will be used to identify the transitions, e.g. $b(2-7)$ instead of $b^{1} \Pi_{u}\left(v^{\prime}=2\right) \longrightarrow X^{1} \Sigma_{g}^{+}\left(v^{\prime \prime}=7\right)$.

The tables are organized as follows:

The first column gives the rotational quantum number $J$ of the electronic ground state. The corresponding $R$ and $P$ branch lines of the $\Sigma \longrightarrow \Sigma$ transitions are listed in the second and third columns and the $R, Q$, and $P$ branch lines of the $\Pi \longrightarrow \Sigma$ transitions are listed in the second, third, and fourth columns. An asterisk is used to identify blended lines that have been assigned more than once, or to indicate, in the case of a missing line, that a feature has been observed within 1.2 and $2 \mathrm{~cm}^{-1}$ from the missing transition.

Because the pressure in the light source is still too high to prevent completely self-absorption at short wavelengths, most of $\left(v^{\prime}, v^{\prime \prime}=0\right)$ bands are missing. However, high- $J$ lines are showing up for several of them. Moreover, a number of emission lines are weakened or missing because they coincide with one or several absorption lines. For many missing lines we have written, in the same column, the name of the molecular absorption lines preventing the emission from being seen. Some missing lines could not be measured because they are overlapped by a strong atomic line which is identified in this column. For the few bands previously reported (Roncin et al. 1984, 1989) one may note slight discrepancies with presently reported wavenumber values. This is because present values are averaged over several measurements, contrary to the previously published data.

\section{References}

Broadfoot A.L., Sandel B.R., Shemansky D.E., et al., 1981, Sci 212,206

Broadfoot A.L., Atreya S. K., Bertaux J.L., et al., 1989, Sci 246,1459 
Roncin J.-Y., Larzillière M., Launay F., 1984, Phys. Rev. Lett. 53, 159

Roncin J.-Y., Launay F., Yoshino K., 1989, J. Molec. Spectrosc. 134, 390

Roncin J.-Y., Subtil J.-L., Launay F., 1998, J. Molec.
Spectrosc. (to be published)

Strobel D.F., Shemansky D.E., 1982, J. Geophys. Res. 87, 1361

Tilford S.G., Wilkinson P.G., 1964, J. Molec. Spectrosc. 12, 1747 